\title{
Dynamic Spectrum Algorithm Based on D2D Communication
}

\author{
Fareha Nizam, Mardeni bin Roslee, Zubaida Yusoff, Abdulraqeb Alhammadi, Khairil Anuar, \\ Hafizal Mohamad
}

\begin{abstract}
Device-to-device (D2D) communication is a concept that promises the overall performance enhancement by allowing direct communication between the devices which are in proximity. The idea of implementing in-band and out-band spectrums together in a D2D assisted mobile users will be relevant to the landscape of the $5 G$ networks. Nevertheless, limited research works are available on efficient transmission of the data when both spectrums are used simultaneously. In this paper, we propose an efficient dynamic spectrum that utilize the licensed and unlicensed bands, based on the distance between the D2D link, in such a way that it selects the best band for establishing the D2D links in the network. The proposed algorithm is based on the distance between the D2D link, where it selects the most efficient band that reduces the interference of the D2D connection and maximizes the network throughput. The simulation results show that the proposed algorithm, using dynamic spectrum, achieves a higher network performance compared with other static spectrums.
\end{abstract}

Keywords-Device to device Communication, Cellular Networks, LTE, LTE-A, Interference management

\section{INTRODUCTION}

Due to the explosive increase of the mobile devices and the limited bandwidth resources, the mobile broadband cannot maintain the high speed of transmission and therefore is pushing the limits of $4 \mathrm{G}$ LTE systems. The increase in the cellular user, results in increase in the workload of the base station. Due to this issue, the new paradigm introduced as an alternative to the conventional cellular network is the device-to-device (D2D) communication, which promises high data rate services for a short distance transmission and boost the LTE's overall performance in the future $5 \mathrm{G}$ and beyond.

Revised Manuscript Received on August 18, 2019

Fareha Nizam, Faculty of Engineering, Multimedia University, Cyberjaya, Malaysia.

Mardeni bin Roslee, Faculty of Engineering, Multimedia University, Cyberjaya, Malaysia.

Zubaida Yusoff, Faculty of Engineering, Multimedia University, Cyberjaya, Malaysia.

Abdulraqeb Alhammadi, Faculty of Engineering, Multimedia University, Cyberjaya, Malaysia.

Khairil Anuar, Faculty of Engineering, Multimedia University, Cyberjaya, Malaysia.

Hafizal Mohamad, Faculty of Engineering, Multimedia University, Cyberjaya, Malaysia.

In D2D communication mode, the transmitting device in the proximity establishes a direct link to the receiving device without using the base station and using only a single link to deliver the data directly. This lower the power consumption as well as conserve the bandwidth resources. However, there are certain challenges that is being faced example power distribution, resource allocation, channel management and energy consumption.

Based on the advantages and the potentials stated above about D2D communication, D2D based transmission technology is used in this paper in order to develop a system to improve the throughput of the D2D network transmission, where it is assumed that each device consist of both the D2D as well as the cellular transmission modes. Additionally, both, in band as well as the out band spectrum is considered, as it is the potentially relevant in the cellular network in the future $5 \mathrm{G}$.

Recently, there have been very narrow research on the usage of in band and out band spectrum, simultaneously. Therefore, this paper investigates this gap using the previous researches on D2D communications.

In this paper, our major contribution is that we proposed a dynamic frequency transmission algorithm in which efficient transmission of data between the transmitting user and the receiving user is achieved, using dynamic frequency allocation technique. The D2D mode has been designed to assign the in band and out band spectrum for the data transmission based on the distance between the D2D link. The simulation results for different network set up are provided which shows that the proposed algorithm provides a higher SINR vs CDF compared to the static spectrum results. Furthermore, analysis and investigation of the dynamic spectrum shows the higher throughput with regards to the distance between the transmitting device and the receiving device.

The remainder of the paper is organized as follows. In the section II, we present the related work done previously. Section III presents the system model and the configurations for the proposed work. Section IV shows and elaborates the simulation results and the analysis. Finally, in Section $\mathrm{V}$, conclusion is given.

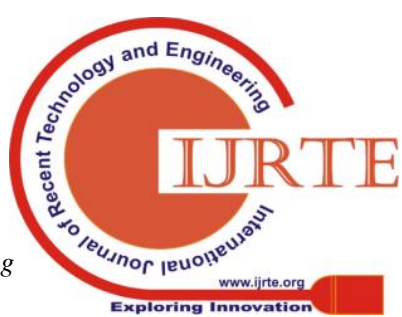




\section{Dynamic Spectrum Algorithm Based on D2D Communication}

\section{RELATED WORK}

Two different mobility managements exist in cellular networks: user equipment (UE) mobility with respect to spectrum and UE mobility with respect to base station (BS). Dynamic Spectrum is more related to spectrum utilization in cognitive radio technology [1-3], whereas mobility with respect to BS maintains the connection between UE and BS, and the UE moves from one BS to another.

In [4] by Asadi et al. the overall and a complete comprehensive survey of D2D communication is provided. Generally, the D2D communication can be categorized into: In-band: where the frequency spectrum is licensed and Out band: where the frequency spectrum for the cellular user is licensed but the frequency spectrum for the D2D transmission is unlicensed.

Till date, most of the work is being done for the in-band and very few for out-band. One of the major issues challenged in the in-band D2D network is the interference control. Doppler et al [5] addressed this issue and proposed to reduce the transmission power as well as the distance between D2D links.

In the paper by [6], the authors used linear interference alignment techniques in order to solve the joint D2D mode selection and mitigate interference, likewise in [7] they maximized the mean throughput of the system and minimized the average delay by using queuing and decision process model. In 2017 [8], the authors proposed a graph theory-based solution using concatenated bi-partite matching (CBM) method in order to enhance the overall performance of D2D in the overall network. Furthermore, this method also mitigates the interference. [9] in this paper, the authors proposed an interference management system with the quality of service (QoS) aware, by applying the graph theory based sub-optimal solution for the power adoption and relay selection.

The advantages and disadvantages of device to device communication varies when it comes to in-band and out-band spectrum. These includes controlling interference, the efficiency of spectral use and the complexity of the implementation of the system. However, very few researches has been done for using both unlicensed and licensed D2D communication. Paper by Asadi et al. [10] and [11] focus on the heuristic solution called 'Floating Band D2D'. These studies mainly exploit the idea of network with both in-band and out-band communication. However, in both works, the transmission at different spectrum was subject to the selection of the spectrum to be utilize, therefore, the overall performance was not affected due to the lack of flexibility in the use of two different bands. Furthermore, in the recent research by Chung et al. [12] the authors proposed an approach to maximize the utilization of the bandwidth, and in and [13], he suggested another approach of reducing the transmission time in a speedy and efficient way, based on the Poisson arrival.

In the paper [14], the author presents a study on signal strength with frequency approaches from $1.8 \mathrm{GHz}$ to $2.2 \mathrm{GHz}$ in indoor environments. It focuses more on mobility of the receiver device, where there will be different distances. In the paper, an USRP (Universal Software Radio Peripheral) device is used as base stations to transmit the desired frequency as well as to receive the signal strength at certain distances in the indoor environment.
However, regardless of all the literature presented above, the overall improvement in the D2D-assisted cellular network using the unlicensed and the licensed bands have to be comprehensively addressed. Therefore, this paper presents the work by using the licensed and the unlicensed bands simultaneously in order to have more efficient transmission. To be more defined, this paper showcases the idea which will enhance the performance of the D2D communication that could eventually improve the applications of D2D. In order to fulfil this, this paper provides overall background and the literature review of the most recent researches., together with the wholesome study of the network model, the detailed algorithm and the performance results of the simulation.

\section{SYSTEM MODEL}

In this work, the simulation was used to evaluate the performance of the proposed dynamic spectrum allocation algorithm for D2D communication. We considered a network system composed of single-cell network. Considering a hexagonal shaped cell with the radius $\mathrm{R}$ and $\mathrm{a}$ single base station located in the middle of the cell. We considered $n$ interfering device and $m$ D2D pair, where $\mathrm{n}>>\mathrm{m}$. We assume only one D2D pair in the cell area. This D2D pair includes a transmitting device ( $D 2 D^{T x}$ ) and receiving device $\left(D 2 D^{R x}\right)$. All the user devices are uniformly distributed within the cell area.

We assumed that all the devices consist of both the cellular mode as well as the D2D mode. Two bands, one unlicensed band and one licensed band is available for the transmission of data., with bandwidth equal to $B_{\text {inband }}$ and $B_{\text {outband }}$ respectively. Furthermore, the cellular mode can only transmit through the licensed band while the D2D mode is able to use either of the two bands. In order to simplify the model, we assume that all the devices in the cell are equipped with only omnidirectional antennas. The set up environment is shown in the Fig. 1.

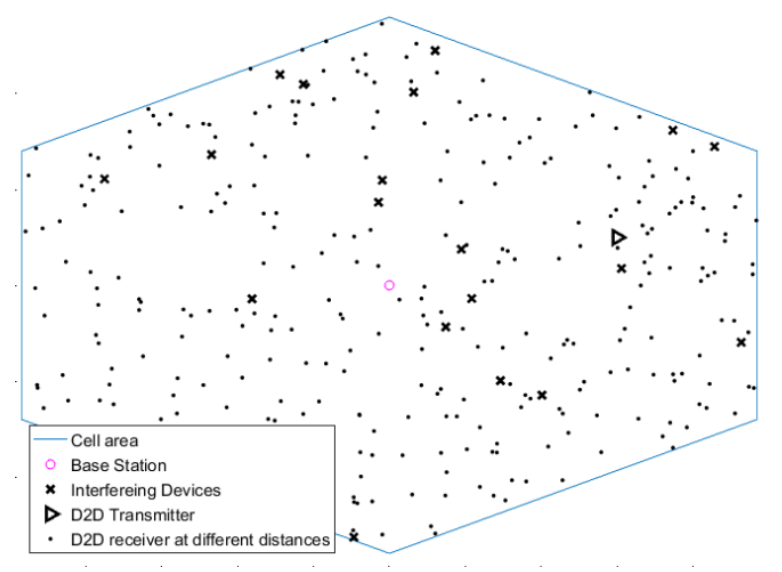

Fig. 1. Simulated Set-up Environment

We ran the simulation 300 times to evaluate the performance according to 300 different distance between the $D 2 D^{T x}$ and $D 2 D^{R x}$. The transmitting power of D2D user ( $P_{T x}^{D 2 D}$ ) and the interfering users $\left(P_{T x}^{I n t}\right)$ users is $24 \mathrm{dBm}$ and $23 \mathrm{dBm}$ respectively. Other 
simulation parameters are shown in the Table 1 . In the proposed algorithm, two different spectrums are allocated to D2D user equipments (UEs) according to the distance between them. If the D2D UEs are located nearby, that is if less than 15m, D2D UEs can use the out-band spectrum.

The out-band spectrum for the system is $\mathrm{mm}$ wave, therefore the system will cause minimum interference for short distances. In case the D2D UEs are located far away from each other, they will be allocated with the in-band spectrum, that is $2 \mathrm{GHz}$. Fig. 4 shows the procedure of how the dynamic spectrum is allocated to the D2D UEs in the proposed algorithm.

TABLE I. PARAMETERS

\begin{tabular}{|c|l|}
\hline Parameters & Values \\
\hline Carrier Frequency - Out-band & $60 \mathrm{GHz}[14]$ \\
\hline Carrier Frequency -In-band [12] & $2.0 \mathrm{GHz}[15]$ \\
\hline Out-band Bandwidth $B_{\text {outband }}$ & $1.5 \mathrm{GHz}[14]$ \\
\hline In-band Bandwidth $B_{\text {inband }}$ & $5 \mathrm{MHz}$ \\
\hline Number of Interfering users & 20 \\
\hline Number of D2D links & 1 \\
\hline Cell Radius & $500 \mathrm{~m}$ \\
\hline Noise power density & $-174 \mathrm{dBm} / \mathrm{Hz}$ \\
\hline$P_{T x}^{D 2 D}$ & $24 \mathrm{dBm}$ \\
\hline$P_{T x}^{\text {Int }}$ & $23 \mathrm{dBm}$ \\
\hline & \\
\hline
\end{tabular}

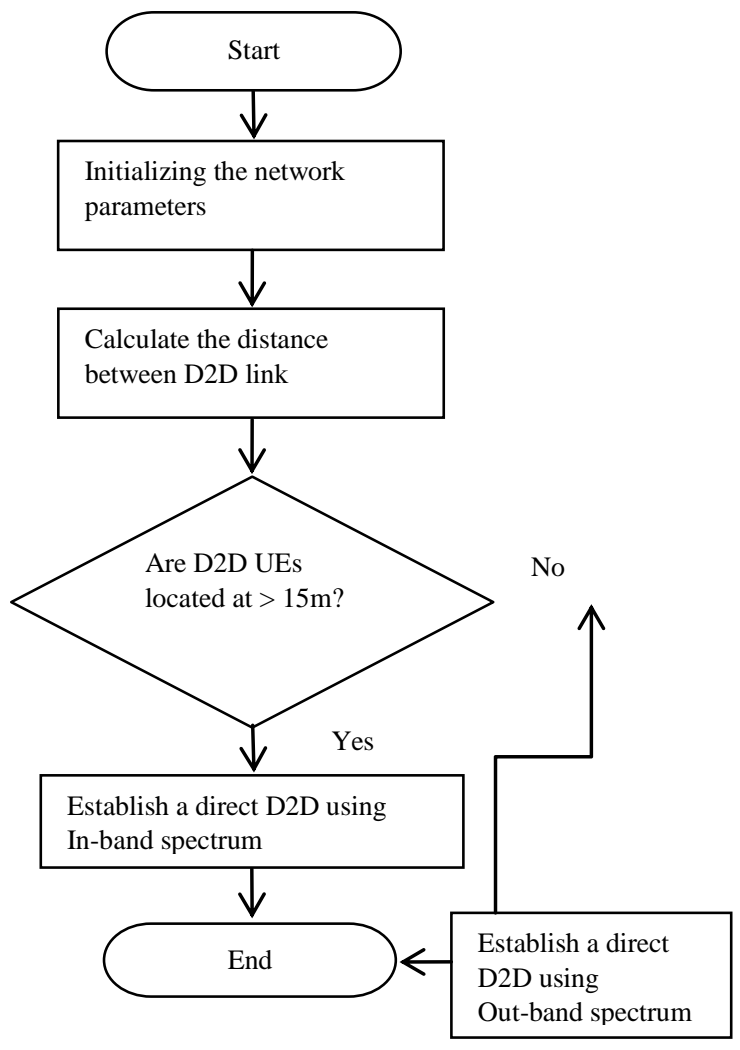

Fig. 2. Flowchart of the procedure of dynamic spectrum D2D UEs
The path-loss are modelled according to the free space, urban and indoor (LOS) models. We apply different path-loss models to D2D UEs for long and short distance as well for the interfering UEs for long and short distances as shown in (1) [1], (2) [16], (3) [16] and (4) [16].

$$
\begin{aligned}
& \bar{\Delta}_{D 2 D_{j, h}^{R}}^{\text {long }}=20 \log _{10}\left(f_{c}[k H z]\right)+20 \log _{10}(d[m])+32.45 \\
& \bar{\Delta}_{D 2 D_{j, h}^{R}}^{\text {short }}=12 \log _{10}\left(f_{c}[k H z]\right)+12 \log _{10}(d[m])+19.47 \\
& \bar{\Delta}_{\text {Int } t_{j, h}^{R}}^{\text {long }}=34 \log _{10}\left(f_{c}[k H z]\right)+34 \log _{10}(d[m])+64.9 \\
& \bar{\Delta}_{\text {shtort }}^{\text {shor }}=20 \log _{10}\left(f_{c}[k H z]\right)+20 \log _{10}(d[m])+32.45
\end{aligned}
$$

(4)

Where $\bar{\Delta}_{D 2 D_{j, h}^{R}}^{\text {long }}$ and $\bar{\Delta}_{D 2 D_{j, h}^{R}}^{\text {short }}$ is the link between the D2D sender and receiver at the long distance and short distance respectively. $\bar{\Delta}_{I n t_{j, h}^{R}}^{\text {long }}$ and $\bar{\Delta}_{I n t_{j, h}^{R}}^{\text {short }}$ is the link between the D2D receiver and the interfering UE at the long and short distance respectively. $d$ represents the distance between a sender and a receiver in meters and the $f_{c}$ is the carrier frequency in $\mathrm{kHz}$.

Now we assume that the strength of the received signal is $\bar{\rho}_{d 2 d}^{\text {long }}$ and $\bar{\rho}_{d 2 d}^{\text {short }}$ from the $j^{\text {th }}$ to the $h^{\text {th }} D 2 D^{T x}$ for the long and short distance respectively. The SINR of the $D 2 D_{i, j, h}^{R}$ is $D 2 D^{R x}$

$$
\begin{gathered}
\gamma_{D 2 D_{i, j, h}^{R}}^{\text {short }}=\frac{R_{D 2 D_{i, j, h}^{\text {short }}}^{\text {short }}}{N_{o}+\sum \bar{\rho}_{\mathrm{int}}} \\
\gamma_{D 2 D_{i, j, h}^{R}}^{\text {long }}=\frac{R_{D 2 D_{i, j, h}}^{\text {long }}}{N_{o}+\sum \bar{\rho}_{\mathrm{int}}^{\text {long }}}
\end{gathered}
$$




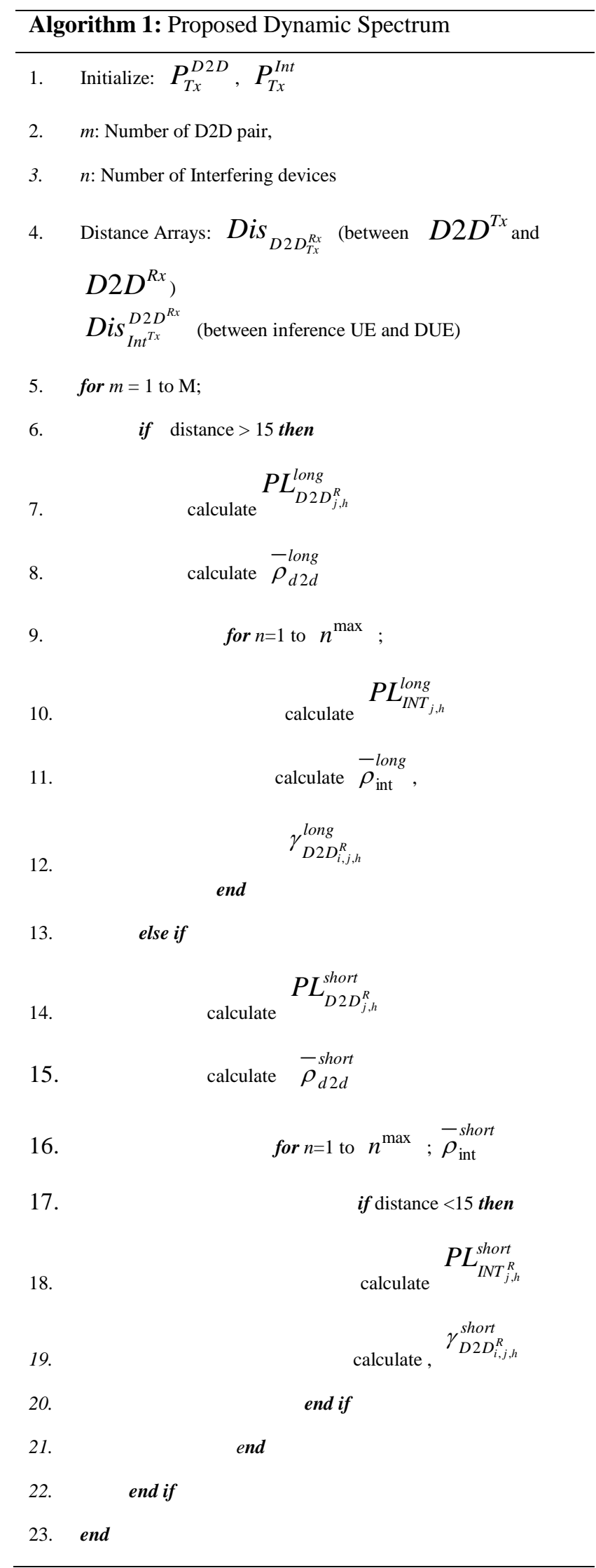

Fig. 3. Proposed Algorithm
$N_{o}$ is the white noise. $\bar{\rho}_{\text {int }}^{\text {long }}$ and $\bar{\rho}_{\text {int }}^{\text {short }}$ is the strength of the interfering signal from the interfering UE to the $D 2 D^{R x}$. We can also analyse the throughput of the $D 2 D_{j, h}^{R}, T_{D 2 D_{i, j, h}^{R}}^{\text {long }}$ and $T_{D 2 D_{i, j, h}^{R}}^{\text {short }}$ by using the Shannon theorem as:

$$
\begin{aligned}
& \underset{D 2 D_{i, j, k}^{R}}{T_{\text {short }}^{\text {sholicensed }}}+10 \log _{2}\left(1+\gamma_{D 2 D_{i, j, k}^{R}}^{\text {short }}\right) \\
& \underset{D_{D 2 D_{i, j, k}^{R}}^{\text {long }}}{T_{\text {licensed }}}+10 \log _{2}\left(1+\gamma_{D 2 D_{i, j, k}^{R}}^{\text {long }}\right)
\end{aligned}
$$

Fig. 3 shows the algorithmic methodology of the proposed system. First, when the data is ready to be transmitted through D2D transmission, the distance between the devices is calculated. The system determines whether the pair can use D2D transmission over unlicensed band or licensed band; in other words, it compares the interference caused at licensed and unlicensed bands. If the distance is greater than $15 \mathrm{~m}$, the system allocates licensed band for the transmission, likewise if the distance is less than $15 \mathrm{~m}$, the unlicensed band is set for the data transmission.

\section{PERFORMANCE ANALYSIS}

This section includes the detailed examination of the evaluation of the performance of the proposed dynamic spectrum transmission algorithm in D2D assisted cellular network, along with the simulations that was performed for different set of UEs. The dynamic proposed algorithm was compared with in-band and out-band spectrum. In this simulation, we consider three performance metrics to evaluate the proposed algorithm: SINR, throughput and interference.

Fig. 4 shows the performance of the proposed algorithm in terms of SINR vs CDF. It provides a comparison of the approach proposed herein with two different approaches using static licensed and unlicensed bands. In the proposed algorithm, the D2D transmission is permitted to use the in-band or the out-band spectrum based on the distance between the transmitting and the receiving device. The results demonstrate that the proposed dynamic spectrum obtains a significant SINR improvement in comparison with the static in-band and out-band spectrum. As the device can select the spectrum based on the distance between the transmitter and the receiver and the SINR between the devices, it mitigates the interference that occurs from the interfering devices surrounding the receiver. Thus, effecting the overall SINR for the specific data transmission. For example, when at CDF $=0.7$; SINR at proposed system is $-8.497 \mathrm{~dB}$, while in-band and out-band spectrums achieve SINR of $-13.92 \mathrm{~dB}$ and $-15.13 \mathrm{~dB}$, respectively.

Furthermore, at the CDF $=0.4$ the SINR of proposed system is -10.79 , while at in-band and out-band spectrum the SINR is -16.49 and $-133.3 \mathrm{db}$ respectively. It can be observed that SINR of the out-band spectrum below 0.7 is below $-120 \mathrm{~dB}$. This shows that using static out-band spectrum mm-wave, the SINR affects due to the interference factor and path-loss between the D2D link due to short wavelength. 
The circle in Fig. 4, shows the part where there is no received power. In the figure our proposed algorithm outperforms basic scheme in terms of SINR.

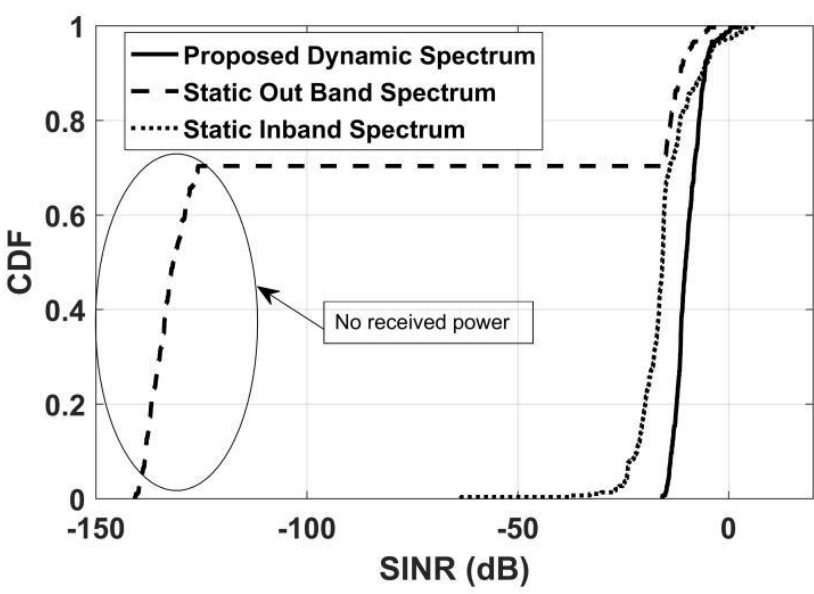

Fig. 4. System SINR CDF compared to Static Spectrum SINR CDF

Fig. 5 shows the throughput of the D2D UE located in the cell area, with respect to the increase in the distance between the D2D link. In static spectrum system, the performance of the user is poor due to the interference and in the out-band spectrum, the path-loss is very high at a certain distance that the receiving power becomes zero. The proposed algorithm allocates the resource according to the distance therefore, the throughput of the users is greatly improved.

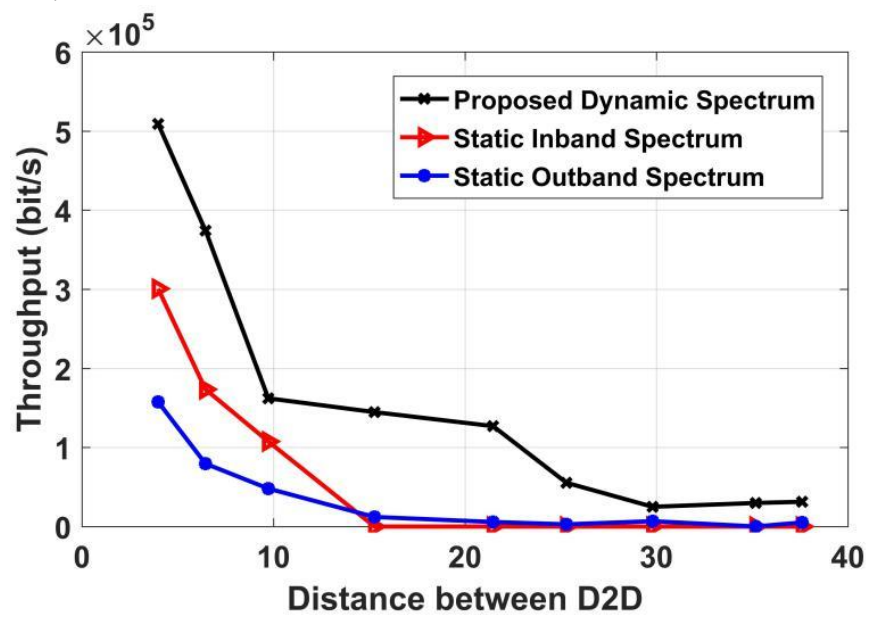

Fig. 5. The overall System throughput for different approaches

Fig. 6 shows the evolution of throughput of the proposed algorithm when the distance between the D2D link is increased. For the performance analysis, the interfering devices for each run was increased from 5 , 10,15 to 20 . When the transmitting power of the D2D transmitter is set as $24 \mathrm{dBm}$, it can be seen, the throughput of the system increases as the number interfering devices decreases. Also, the throughput of the overall system decreases as the distance between the D2D link is increased. The proposed algorithm can sustain more than 5 Mbps at the distance of $3 \mathrm{~m}$, when the total interfering devices are 5 , while the system can sustain about $4 \mathrm{Mbps}$ at the same distance when the total interfering devices is 10 .

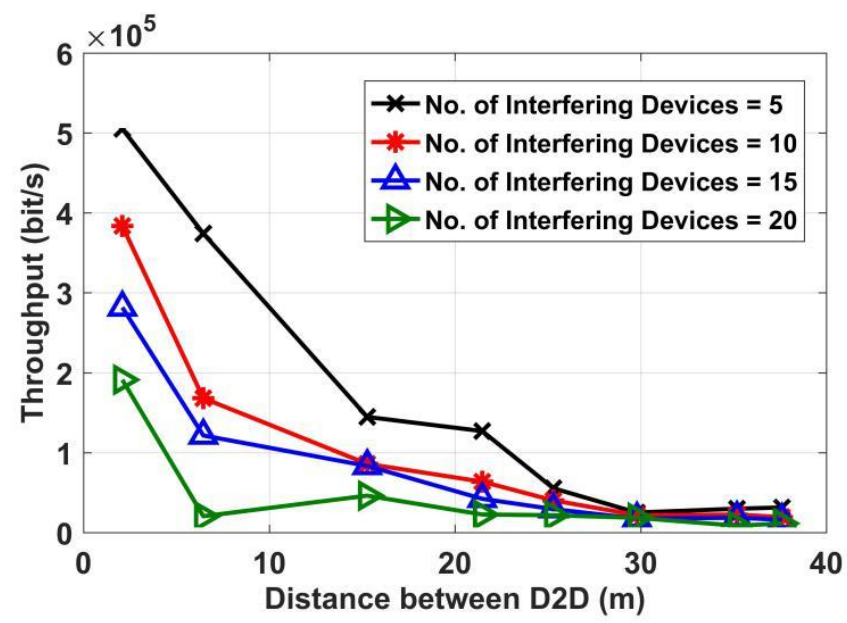

Fig. 6. Throughput analysis of proposed algorithm

Fig. 7 shows the interference induced from the interfering devices with different distance between the D2 D2D transmitter and receiver. It indicates the distance between the devices and the number of interfering devices influences the overall interference of the system heavily. When the $\mathrm{D} 2 \mathrm{D}$ receiver gets closer to the $\mathrm{D} 2 \mathrm{D}$ transmitter, the interference will be lower and therefore the throughput is more. This phenomenon is mainly because of the path-loss between the long distance D2D link and the transmission signals from the interfering devices.

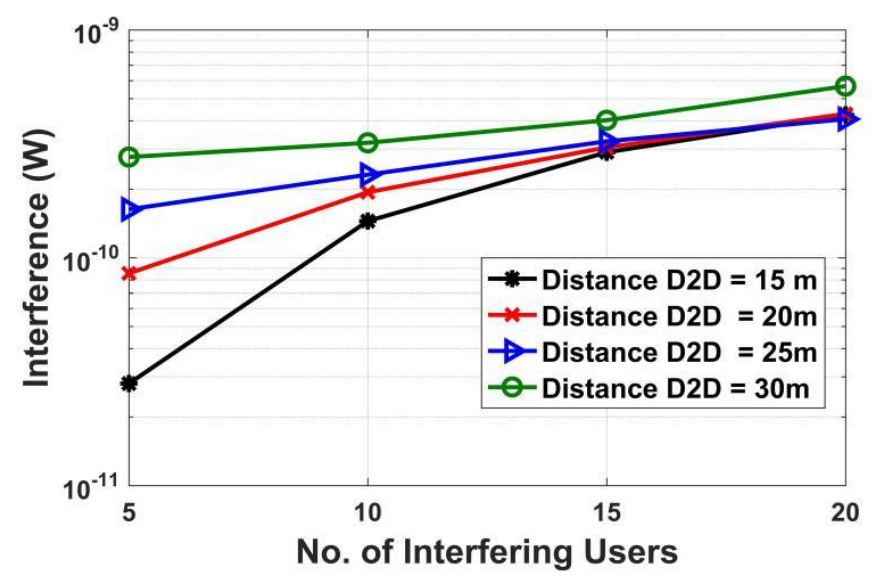

Fig. 7. Interference versus No. of interfering devices with different D2D distance

\section{ACKNOWLEDGMENT}

This research was supported in part by Fundamental Research Grant Scheme (MMUE/170017), Ministry of Higher Education, Malaysia.

Published By: 


\section{Dynamic Spectrum Algorithm Based on D2D Communication}

\section{REFERENCES}

1. A. Alhammadi, M. Roslee and M. Y. Alias, "Analysis of spectrum handoff schemes in cognitive radio network using particle swarm optimization," 2016 IEEE 3rd International Symposium on Telecommunication Technologies (ISTT), Kuala Lumpur, 2016, pp. 103- 107.

2. A. Alhammadi, M. Roslee and M. Y. Alias, "Fuzzy logic based negotiation approach for spectrum handoff in cognitive radio network," $2016 \quad$ IEEE 3rd International Symposium on Telecommunication Technologies (ISTT), Kuala Lumpur, 2016, pp. 120- 124.

3. R. Mardeni, A. Abdulrab, M. Y. A, N. P. Khairil, "Efficient handoff spectrum scheme using fuzzy decision making in cognitive radio system", 2017 3rd International Conference on Frontiers of Signal Processing (ICFSP), 2017.

4. A. Asadi, Q. Wang, and V. Mancuso, "A survey on device-to-device communication in cellular networks," IEEE Commun. Surveys Tuts., vol. 16, no. 4, pp. 1801-1819, Nov. 2014

5. K. Doppler, M. Rinne, C. Wijting, C. Ribeiro, and K. Hugl, "Device-todevice communication as an underlay to LTE-Advanced networks," IEEE Commun. Mag., vol. 47, no. 12, pp. 42-49, Dec. 2009

6. H.-J. Chou and R. Y. Chang, "Joint mode selection and interference management in device-to-device communications underlaid MIMO cellular networks," IEEE Trans. Wireless Commun., vol. 16, no. 2, pp. 1120-1134, Feb. 2017

7. P. Cheng, L. Deng, H. Yu, Y. Xu, and H. Wang, "Resource allocation for cognitive networks with D2D communication: An evolutionary approach," in Proc. IEEE Wireless Commun. Netw. Conf. (WCNC), Shanghai, China, 2012, pp. 2671-2676

8. A. Celik, R. M. Radaydeh, F. S. Al-Qahtani, and M.-S. Alouini, "Resource allocation and interference management for D2D-enabled DL/UL decoupled Het-Nets," IEEE Access, vol. 5, pp. 22735-22749, 2017.s

9. T. Yang, X. Cheng, X. Shen, S. Chen, and L. Yang, "QoS-aware interference management for vehicular D2D relay networks," J. Commun. Inf. Netw., vol. 2, no. 2, pp. 75-90, 2017

10. Asadi, P. Jacko, and V. Mancuso, "Modeling D2D communications with LTE and WiFi," ACM SIGMETRICS Perform. Eval. Rev., vol. 42, no. 2, pp. 55-57, 2014.

11. A. Asadi, V. Mancuso, and P. Jacko, "Floating band D2D: exploring and exploiting the potentials of adaptive D2D-enabled networks," in Proc. IEEE Int. Symp. World Wireless, Mobile Multimedia Netw., Boston, MA, USA, 2015, pp. 1-9

12. Y.-L. Chung and J.-A. Wu, "A simple yet effective approach for resource allocation in outband device-to-device communication," in Proc. 4th IEEE Int. Conf. Future Gener. Commun. Technol., Luton, U.K., Jul. 2015, pp. 1-3

13. Y.-L. Chung, "Fast and efficient transmission in D2D-assisted cellular networks," in Proc. 15th IEEE Wireless Telecommun. Symp., London, U.K., Apr. 2016, pp. 1-5

14. Mardeni R., A. Alhammadi, F.Farhana Ruslan, K. Anuar, M.Y.Alias, H.Mohamad, (Nov 2017), Analysis of LTE-A Signal Strength in Indoor Mobility Environment, Advanced Science Letters Journal, Vol.23, No.11, pp11355-11359, ISSN: 1936-6612, 1936-731

15. Zhang, Z., Wang, C., Yu, H., Wang, M., \& Sun, S. (2018). Power Optimization Assisted Interference Management for D2D Communications in mmWave Networks. IEEE Access

16. Belleschi, Marco, Gabor Fodor, and Andrea Abrardo. "Performance analysis of a distributed resource allocation scheme for D2D communications." In GLOBECOM Workshops (GC Wkshps), 2011 IEEE, pp. 358-362. IEEE, 2011

17. Al-Hourani, Akram, Sathyanarayanan Chandrasekharan, and Sithamparanathan Kandeepan. "Path loss study for millimeter wave device-to-device communications in urban environment." In Communications Workshops (ICC), 2014 IEEE International Conference on, pp. 102-107. IEEE, 2014

\section{AUTHORS PROFILE}

Fareha Nizam earned her B. in Electrical and Electronics Engineering from Nilai University, Malaysia in 2017. She is currently serving as a Graduate

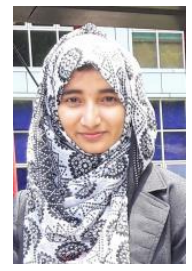
Research Assistant in Multimedia University in Centre of Wireless Communication while pursuing M.Eng.Science from Multimedia University, Malaysia. She received "Best Project Award" for her Final Year Project in 2017 and silver medal at RICES 2018 for her work in device to device communication in $4 \mathrm{G} / 5 \mathrm{G}$ network. She is an executive committee member of WIE IEEE MMU and IEEE MMU. She is also a member of IEEE since 2017 and a member of a number of international committees and societies.

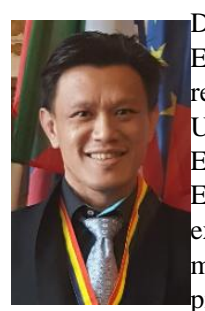

Dr.Mardeni is an Associate Professor under Faculty of Engineering, Multimedia University, Cyberjaya. He is a registered Chartered Engineer with Engineering Council United Kingdom, and Member with The Institution of Engineering and Technology (IET), UK. As a Chartered Engineer, he bring a diversified range of engineering experience in design \& development and engineering management. His experiences include the consultation, professional institution and academic sectors. $\mathrm{He}$ is a Senior Member of IEEE. His current research interests are 5G/6G telecommunication, satellite, IoT and radar. $\mathrm{He}$ is a chair of IEEE Comsoc/VTS, Malaysia and head of Malaysian Radar \& Navigations Interest Group (MyRaN ig) which is under Malaysian Society for Engineering \& Technology (MY SET), which is for recognized and selected members in professional organization, networking and interaction with like-minded multidisciplinary professionals from public and private sectors as well as the international platforms in the $21^{\text {st }}$ century. In Multimedia University, he has been appointed as a President of Multimedia University Mesra and Chairman of Centre for Wireless Technology. His contributions to academic and the engineering professional over the years have earned him recognitions nationally and international, he has awarded 24 international/local awards and been awarded the University Excellent Researcher Award for 2016 and 2018, respectively and Prestigious European Award for year 2018, which is the only one from Malaysia.

Dr Zubaida Yusoff holds the position of a Senior Lecturer at the Faculty of

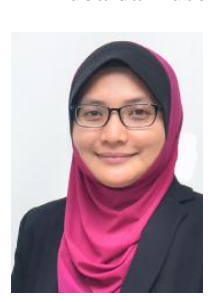
Engineering, Multimedia University. She received her B.Sc. in Electrical and Computer Engineering (cum laude with distinction) and M.Sc. in Electrical Engineering from The Ohio State University, USA in 2000 and 2002 respectively. She worked with Telekom Malaysia

International Network Operation in 2002 before she joined Multimedia University in 2004. She continued her studies at Cardiff University, Wales, UK in 2008 and received $\mathrm{Ph} . \mathrm{D}$ degree in 2012. Dr Zubaida has presented technical papers at conference nationally and internationally. One of her conference papers has received "Honorable Mention" for the Student Paper Competition at the International Microwave Symposium, USA in 2011. She has authored/co-authored more than 25 journals and conference papers. Her teaching and research focuses in the area of Microelectronics, Analog/Mixed Signal RF Circuit Design, Power Amplifier Design, Antenna and 5G Communications. 


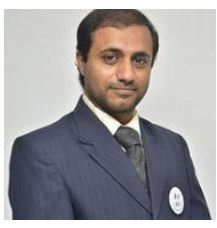

Abdulraqeb Alhammadi received the B. Eng. in Electronic majoring in telecommunications and M.S degree in wireless communication from Multimedia University, Malaysia, in 2011 and 2015 respectively. He is currently serving as a research scholar at Multimedia University since 2012. He is the author of more than 20 papers in international journals and conferences. His main research interests are in heterogeneous networks, mobility management, device-to-device communication, cognitive radio networks, localization, propagation modelling. $\mathrm{He}$ is a member of professional institutes and societies such as IEEE, IEICE, IACSIT and IAENG. He is also a member of more than 20 program committees at international conferences and workshops.

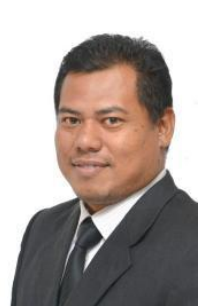

Mr. Khairil Bin Anuar is a lecturer in the Faculty of Engineering at Multimedia University (MMU), Malaysia. His research involved in Computer Networking, Mobile and Wireless Communications, Radio Defined Networks, Software Defined Networks and Internet of Things. He serves in various committee for faculty and university including Staff Welfare Committee, ITP committee, LMC committee, Garden Tea Party Committee, Alumni Homecoming Committee, Sports Carnival Committee etc. He is also well known in his research area and actively involved in the IEEE Malaysia ComSoc/VTS Joint Committee. He also helps to coordinate most of the events, workshop and conferences. IEEE ICC 2016 and IEEE VTS 2019 are two flagship conferences that he manage. Other local conferences are MICC, ISTT and SCOReD. During his involvements, ComSoc/VTS had won various awards locally, Asia Pacific and Internationally.

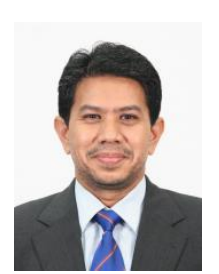

Hafizal Mohamad earned a BEng (First Class Honours) and a $\mathrm{PhD}$ degree in electronic engineering from University of Southampton, UK. He is the recipient of the ASEAN Outstanding Scientist and Technologist Award (AOSTA). Currently, he is Senior Staff Researcher at Corporate Technology Division, MIMOS Berhad, where he involves in R\&D and commercial projects related to Internet of Things and mesh networks. He is the co-inventor of 36 filed patents and 9 granted patents in the field of wireless communication. He has published over 100 papers in international journals and conference proceedings. Prior to MIMOS, he was a faculty member at the Multimedia University, Malaysia. He served as a visiting fellow at NICT, Japan in 2005. He is a Registered Professional Engineer with the Board of Engineers Malaysia and a Senior Member of the Institution of Institute of Electrical and Electronics Engineers (IEEE), USA. He has served in various leadership roles in the IEEE, including the Vice Chair for IEEE Malaysia Section (2013) and the Chair for IEEE ComSoc/VT Chapter (2009-11). He was the Conference Operation Chair of the IEEE ICC 2016 and the Technical Program Chair for the IEEE VTC2019. 